Network Working Group

Request for Comments: 2581

Obsoletes: 2001

Category: Standards Track
M. Allman

NASA Glenn/Sterling Software

V. Paxson

ACIRI / ICSI

W. Stevens Consultant April 1999

\title{
TCP Congestion Control
}

Status of this Memo

This document specifies an Internet standards track protocol for the Internet community, and requests discussion and suggestions for improvements. Please refer to the current edition of the "Internet Official Protocol Standards" (STD 1) for the standardization state and status of this protocol. Distribution of this memo is unlimited.

Copyright Notice

Copyright (C) The Internet Society (1999). All Rights Reserved.

Abstract

This document defines $\mathrm{TCP}^{\prime} \mathrm{s}$ four intertwined congestion control algorithms: slow start, congestion avoidance, fast retransmit, and fast recovery. In addition, the document specifies how TCP should begin transmission after a relatively long idle period, as well as discussing various acknowledgment generation methods.

1. Introduction

This document specifies four TCP [Pos81] congestion control algorithms: slow start, congestion avoidance, fast retransmit and fast recovery. These algorithms were devised in [Jac88] and [Jac90]. Their use with TCP is standardized in [Bra89].

This document is an update of [Ste97]. In addition to specifying the congestion control algorithms, this document specifies what TCP connections should do after a relatively long idle period, as well as specifying and clarifying some of the issues pertaining to TCP ACK generation.

Note that [Ste94] provides examples of these algorithms in action and [WS95] provides an explanation of the source code for the BSD implementation of these algorithms. 
This document is organized as follows. Section 2 provides various definitions which will be used throughout the document. Section 3 provides a specification of the congestion control algorithms. Section 4 outlines concerns related to the congestion control algorithms and finally, section 5 outlines security considerations.

The key words "MUST", "MUST NOT", "REQUIRED", "SHALL", "SHALL NOT", "SHOULD", "SHOULD NOT", "RECOMMENDED", "MAY", and "OPTIONAL" in this document are to be interpreted as described in [Bra97].

2. Definitions

This section provides the definition of several terms that will be used throughout the remainder of this document.

SEGMENT :

A segment is ANY TCP/IP data or acknowledgment packet (or both).

SENDER MAXIMUM SEGMENT SIZE (SMSS): The SMSS is the size of the largest segment that the sender can transmit. This value can be based on the maximum transmission unit of the network, the path MTU discovery [MD90] algorithm, RMSS (see next item), or other factors. The size does not include the TCP/IP headers and options.

RECEIVER MAXIMUM SEGMENT SIZE (RMSS): The RMSS is the size of the largest segment the receiver is willing to accept. This is the value specified in the MSS option sent by the receiver during connection startup. Or, if the MSS option is not used, 536 bytes [Bra89]. The size does not include the TCP/IP headers and options.

FULL-SIZED SEGMENT: A segment that contains the maximum number of data bytes permitted (i.e., a segment containing SMSS bytes of data).

RECEIVER WINDOW (rwnd) The most recently advertised receiver window.

CONGESTION WINDOW (cwnd): A TCP state variable that limits the amount of data a TCP can send. At any given time, a TCP MUST NOT send data with a sequence number higher than the sum of the highest acknowledged sequence number and the minimum of cwnd and rwind.

INITIAL WINDOW (IW): The initial window is the size of the sender's congestion window after the three-way handshake is completed. 
LOSS WINDOW (LW): The loss window is the size of the congestion window after a TCP sender detects loss using its retransmission timer.

RESTART WINDOW (RW): The restart window is the size of the congestion window after a TCP restarts transmission after an idle period (if the slow start algorithm is used; see section 4.1 for more discussion).

FLIGHT SIZE: The amount of data that has been sent but not yet acknowledged.

\section{Congestion Control Algorithms}

This section defines the four congestion control algorithms: slow start, congestion avoidance, fast retransmit and fast recovery, developed in [Jac88] and [Jac90]. In some situations it may be beneficial for a TCP sender to be more conservative than the algorithms allow, however a TCP MUST NOT be more aggressive than the following algorithms allow (that is, MUST NOT send data when the value of cwnd computed by the following algorithms would not allow the data to be sent).

\subsection{Slow Start and Congestion Avoidance}

The slow start and congestion avoidance algorithms MUST be used by a TCP sender to control the amount of outstanding data being injected into the network. To implement these algorithms, two variables are added to the TCP per-connection state. The congestion window (cwnd) is a sender-side limit on the amount of data the sender can transmit into the network before receiving an acknowledgment ( $A C K)$, while the receiver's advertised window (rwnd) is a receiver-side limit on the amount of outstanding data. The minimum of cwnd and rwnd governs data transmission.

Another state variable, the slow start threshold (ssthresh), is used to determine whether the slow start or congestion avoidance algorithm is used to control data transmission, as discussed below.

Beginning transmission into a network with unknown conditions requires TCP to slowly probe the network to determine the available capacity, in order to avoid congesting the network with an inappropriately large burst of data. The slow start algorithm is used for this purpose at the beginning of a transfer, or after repairing loss detected by the retransmission timer. 
IW, the initial value of cwnd, MUST be less than or equal to $2 *$ SMSS bytes and MUST NOT be more than 2 segments.

We note that a non-standard, experimental TCP extension allows that a TCP MAY use a larger initial window (IW), as defined in equation 1 [AFP98]:

$\mathrm{IW}=\min (4 * \mathrm{SMSS}, \max (2 * \mathrm{SMSS}, 4380$ bytes $))$

With this extension, a TCP sender MAY use a 3 or 4 segment initial window, provided the combined size of the segments does not exceed 4380 bytes. We do NOT allow this change as part of the standard defined by this document. However, we include discussion of (1) in the remainder of this document as a guideline for those experimenting with the change, rather than conforming to the present standards for TCP congestion control.

The initial value of ssthresh MAY be arbitrarily high (for example, some implementations use the size of the advertised window), but it may be reduced in response to congestion. The slow start algorithm is used when cwnd < ssthresh, while the congestion avoidance algorithm is used when cwnd > ssthresh. When cwnd and ssthresh are equal the sender may use either slow start or congestion avoidance.

During slow start, a TCP increments cwnd by at most SMSS bytes for each ACK received that acknowledges new data. Slow start ends when cwnd exceeds ssthresh (or, optionally, when it reaches it, as noted above) or when congestion is observed.

During congestion avoidance, cwnd is incremented by 1 full-sized segment per round-trip time (RTT). Congestion avoidance continues until congestion is detected. One formula commonly used to update cwnd during congestion avoidance is given in equation 2:

$$
\text { Cwnd }+=\text { SMSS*SMSS } / \text { Cwnd }
$$

This adjustment is executed on every incoming non-duplicate ACK. Equation (2) provides an acceptable approximation to the underlying principle of increasing cwnd by 1 full-sized segment per RTT. (Note that for a connection in which the receiver acknowledges every data segment, (2) proves slightly more aggressive than 1 segment per RTT, and for a receiver acknowledging every-other packet, (2) is less aggressive.) 
Implementation Note: Since integer arithmetic is usually used in TCP implementations, the formula given in equation 2 can fail to increase cwnd when the congestion window is very large (larger than SMSS*SMSS). If the above formula yields 0 , the result SHOULD be rounded up to 1 byte.

Implementation Note: older implementations have an additional additive constant on the right-hand side of equation (2). This is incorrect and can actually lead to diminished performance [PAD+98].

Another acceptable way to increase cwnd during congestion avoidance is to count the number of bytes that have been acknowledged by ACKs for new data. (A drawback of this implementation is that it requires maintaining an additional state variable.) When the number of bytes acknowledged reaches cwnd, then cwnd can be incremented by up to SMSS bytes. Note that during congestion avoidance, cwnd MUST NOT be increased by more than the larger of either 1 full-sized segment per RTT, or the value computed using equation 2 .

Implementation Note: some implementations maintain cwnd in units of bytes, while others in units of full-sized segments. The latter will find equation (2) difficult to use, and may prefer to use the counting approach discussed in the previous paragraph.

When a TCP sender detects segment loss using the retransmission timer, the value of ssthresh MUST be set to no more than the value given in equation 3 :

ssthresh $=\max ($ FlightSize / 2, 2*SMSS)

As discussed above, Flightsize is the amount of outstanding data in the network.

Implementation Note: an easy mistake to make is to simply use cwnd, rather than FlightSize, which in some implementations may incidentally increase well beyond rwnd.

Furthermore, upon a timeout cwnd MUST be set to no more than the loss window, LW, which equals 1 full-sized segment (regardless of the value of IW). Therefore, after retransmitting the dropped segment the TCP sender uses the slow start algorithm to increase the window from 1 full-sized segment to the new value of ssthresh, at which point congestion avoidance again takes over. 


\subsection{Fast Retransmit/Fast Recovery}

A TCP receiver SHOULD send an immediate duplicate ACK when an outof-order segment arrives. The purpose of this ACK is to inform the sender that a segment was received out-of-order and which sequence number is expected. From the sender's perspective, duplicate ACKs can be caused by a number of network problems. First, they can be caused by dropped segments. In this case, all segments after the dropped segment will trigger duplicate ACKs. Second, duplicate ACKs can be caused by the re-ordering of data segments by the network (not a rare event along some network paths [Pax97]). Finally, duplicate ACKs can be caused by replication of ACK or data segments by the network. In addition, a TCP receiver SHOULD send an immediate ACK when the incoming segment fills in all or part of a gap in the sequence space. This will generate more timely information for a sender recovering from a loss through a retransmission timeout, a fast retransmit, or an experimental loss recovery algorithm, such as NewReno [FH98].

The TCP sender SHOULD use the "fast retransmit" algorithm to detect and repair loss, based on incoming duplicate ACKs. The fast retransmit algorithm uses the arrival of 3 duplicate ACKs (4 identical ACKs without the arrival of any other intervening packets) as an indication that a segment has been lost. After receiving 3 duplicate ACKs, TCP performs a retransmission of what appears to be the missing segment, without waiting for the retransmission timer to expire.

After the fast retransmit algorithm sends what appears to be the missing segment, the "fast recovery" algorithm governs the transmission of new data until a non-duplicate ACK arrives. The reason for not performing slow start is that the receipt of the duplicate ACKs not only indicates that a segment has been lost, but also that segments are most likely leaving the network (although a massive segment duplication by the network can invalidate this conclusion). In other words, since the receiver can only generate a duplicate ACK when a segment has arrived, that segment has left the network and is in the receiver's buffer, so we know it is no longer consuming network resources. Furthermore, since the ACK "clock" [Jac88] is preserved, the TCP sender can continue to transmit new segments (although transmission must continue using a reduced cwnd).

The fast retransmit and fast recovery algorithms are usually implemented together as follows.

1. When the third duplicate ACK is received, set ssthresh to no more than the value given in equation 3 . 
2. Retransmit the lost segment and set cwnd to ssthresh plus 3*SMSS. This artificially "inflates" the congestion window by the number of segments (three) that have left the network and which the receiver has buffered.

3. For each additional duplicate ACK received, increment cwnd by SMSS. This artificially inflates the congestion window in order to reflect the additional segment that has left the network.

4. Transmit a segment, if allowed by the new value of cwnd and the receiver's advertised window.

5. When the next ACK arrives that acknowledges new data, set cwnd to ssthresh (the value set in step 1). This is termed "deflating" the window.

This ACK should be the acknowledgment elicited by the retransmission from step 1, one RTT after the retransmission (though it may arrive sooner in the presence of significant outof-order delivery of data segments at the receiver). Additionally, this ACK should acknowledge all the intermediate segments sent between the lost segment and the receipt of the third duplicate ACK, if none of these were lost.

Note: This algorithm is known to generally not recover very efficiently from multiple losses in a single flight of packets [FF96]. One proposed set of modifications to address this problem can be found in [FH98].

4. Additional Considerations

\subsection{Re-starting Idle Connections}

A known problem with the TCP congestion control algorithms described above is that they allow a potentially inappropriate burst of traffic to be transmitted after $\mathrm{TCP}$ has been idle for a relatively long period of time. After an idle period, TCP cannot use the ACK clock to strobe new segments into the network, as all the ACKs have drained from the network. Therefore, as specified above, TCP can potentially send a cwnd-size line-rate burst into the network after an idle period.

[Jac88] recommends that a TCP use slow start to restart transmission after a relatively long idle period. Slow start serves to restart the ACK clock, just as it does at the beginning of a transfer. This mechanism has been widely deployed in the following manner. When TCP has not received a segment for more than one retransmission timeout, cwnd is reduced to the value of the restart window (RW) before 
transmission begins.

For the purposes of this standard, we define RW = IW.

We note that the non-standard experimental extension to TCP defined in [AFP98] defines $R W=\min (I W$, cWnd), with the definition of IW adjusted per equation (1) above.

Using the last time a segment was received to determine whether or not to decrease cwnd fails to deflate cwnd in the common case of persistent HTTP connections [HTH98]. In this case, a WWW server receives a request before transmitting data to the WWW browser. The reception of the request makes the test for an idle connection fail, and allows the TCP to begin transmission with a possibly

inappropriately large cwnd.

Therefore, a TCP SHOULD set cwnd to no more than RW before beginning transmission if the TCP has not sent data in an interval exceeding the retransmission timeout.

\subsection{Generating Acknowledgments}

The delayed ACK algorithm specified in [Bra89] SHOULD be used by a TCP receiver. When used, a TCP receiver MUST NOT excessively delay acknowledgments. Specifically, an ACK SHOULD be generated for at least every second full-sized segment, and MUST be generated within $500 \mathrm{~ms}$ of the arrival of the first unacknowledged packet.

The requirement that an ACK "SHOULD" be generated for at least every second full-sized segment is listed in [Bra89] in one place as a SHOULD and another as a MUST. Here we unambiguously state it is a SHOULD. We also emphasize that this is a SHOULD, meaning that an implementor should indeed only deviate from this requirement after careful consideration of the implications. See the discussion of "Stretch ACK violation" in [PAD+98] and the references therein for a discussion of the possible performance problems with generating ACKs less frequently than every second full-sized segment.

In some cases, the sender and receiver may not agree on what constitutes a full-sized segment. An implementation is deemed to comply with this requirement if it sends at least one acknowledgment every time it receives $2 *$ RMSS bytes of new data from the sender, where RMSS is the Maximum Segment Size specified by the receiver to the sender (or the default value of 536 bytes, per [Bra89], if the receiver does not specify an MSS option during connection establishment). The sender may be forced to use a segment size less than RMSS due to the maximum transmission unit (MTU), the path MTU discovery algorithm or other factors. For instance, consider the 
case when the receiver announces an RMSS of $\mathrm{X}$ bytes but the sender ends up using a segment size of $Y$ bytes $(Y<X)$ due to path MTU discovery (or the sender's MTU size). The receiver will generate stretch ACKs if it waits for $2 * X$ bytes to arrive before an ACK is sent. Clearly this will take more than 2 segments of size y bytes. Therefore, while a specific algorithm is not defined, it is desirable for receivers to attempt to prevent this situation, for example by acknowledging at least every second segment, regardless of size. Finally, we repeat that an ACK MUST NOT be delayed for more than 500 ms waiting on a second full-sized segment to arrive.

Out-of-order data segments SHOULD be acknowledged immediately, in order to accelerate loss recovery. To trigger the fast retransmit algorithm, the receiver SHOULD send an immediate duplicate ACK when it receives a data segment above a gap in the sequence space. To provide feedback to senders recovering from losses, the receiver SHOULD send an immediate ACK when it receives a data segment that fills in all or part of a gap in the sequence space.

A TCP receiver MUST NOT generate more than one ACK for every incoming segment, other than to update the offered window as the receiving application consumes new data [page 42, Pos81][Cla82].

\subsection{Loss Recovery Mechanisms}

A number of loss recovery algorithms that augment fast retransmit and fast recovery have been suggested by TCP researchers. While some of these algorithms are based on the TCP selective acknowledgment (SACK) option [MMFR96], such as [FF96, MM96a,MM96b], others do not require SACKS [Hoe96,FF96, FH98]. The non-SACK algorithms use "partial acknowledgments" (ACKs which cover new data, but not all the data outstanding when loss was detected) to trigger retransmissions. While this document does not standardize any of the specific algorithms that may improve fast retransmit/fast recovery, these enhanced algorithms are implicitly allowed, as long as they follow the general principles of the basic four algorithms outlined above.

Therefore, when the first loss in a window of data is detected, ssthresh MUST be set to no more than the value given by equation (3). Second, until all lost segments in the window of data in question are repaired, the number of segments transmitted in each RTT MUST be no more than half the number of outstanding segments when the loss was detected. Finally, after all loss in the given window of segments has been successfully retransmitted, cwnd MUST be set to no more than ssthresh and congestion avoidance MUST be used to further increase cwnd. Loss in two successive windows of data, or the loss of a retransmission, should be taken as two indications of congestion and, therefore, cwnd (and ssthresh) MUST be lowered twice in this case. 
The algorithms outlined in [Hoe96, FF96, MM96a, MM6b] follow the principles of the basic four congestion control algorithms outlined in this document.

\section{Security Considerations}

This document requires a TCP to diminish its sending rate in the presence of retransmission timeouts and the arrival of duplicate acknowledgments. An attacker can therefore impair the performance of a TCP connection by either causing data packets or their acknowledgments to be lost, or by forging excessive duplicate acknowledgments. Causing two congestion control events back-to-back will often cut ssthresh to its minimum value of 2 *SMSS, causing the connection to immediately enter the slower-performing congestion avoidance phase.

The Internet to a considerable degree relies on the correct implementation of these algorithms in order to preserve network stability and avoid congestion collapse. An attacker could cause TCP endpoints to respond more aggressively in the face of congestion by forging excessive duplicate acknowledgments or excessive acknowledgments for new data. Conceivably, such an attack could drive a portion of the network into congestion collapse.

6. Changes Relative to RFC 2001

This document has been extensively rewritten editorially and it is not feasible to itemize the list of changes between the two documents. The intention of this document is not to change any of the recommendations given in RFC 2001, but to further clarify cases that were not discussed in detail in 2001. Specifically, this document suggests what TCP connections should do after a relatively long idle period, as well as specifying and clarifying some of the issues pertaining to TCP ACK generation. Finally, the allowable upper bound for the initial congestion window has also been raised from one to two segments.

Acknowledgments

The four algorithms that are described were developed by Van Jacobson.

Some of the text from this document is taken from "TCP/IP Illustrated, Volume 1: The Protocols" by W. Richard Stevens (Addison-Wesley, 1994) and "TCP/IP Illustrated, Volume 2: The Implementation" by Gary R. Wright and W. Richard Stevens (AddisonWesley, 1995). This material is used with the permission of Addison-Wesley. 
Neal Cardwell, Sally Floyd, Craig Partridge and Joe Touch contributed a number of helpful suggestions.

References

[AFP98] Allman, M., Floyd, S. and C. Partridge, "Increasing TCP'S Initial Window Size, RFC 2414, September 1998.

[Bra89] Braden, R., "Requirements for Internet Hosts -Communication Layers", STD 3, RFC 1122, October 1989.

[Bra97] Bradner, S., "Key words for use in RFCs to Indicate Requirement Levels", BCP 14, RFC 2119, March 1997.

[Cla82] Clark, D., "Window and Acknowledgment Strategy in TCP", RFC 813, July 1982 .

[FF96] Fall, K. and S. Floyd, "Simulation-based Comparisons of Tahoe, Reno and SACK TCP", Computer Communication Review, July 1996. ftp://ftp.ee.lbl.gov/papers/sacks.ps.z.

[FH98] Floyd, S. and T. Henderson, "The NewReno Modification to TCP's Fast Recovery Algorithm", RFC 2582, April 1999.

[Flo94] Floyd, S., "TCP and Successive Fast Retransmits. Technical report", October 1994 . ftp://ftp.ee.lbl.gov/papers/fastretrans.ps.

[Hoe96] Hoe, J., "Improving the Start-up Behavior of a Congestion Control Scheme for TCP", In ACM SIGCOMM, August 1996.

[HTH98] Hughes, A., Touch, J. and J. Heidemann, "Issues in TCP Slow-Start Restart After Idle", Work in Progress.

[Jac88] Jacobson, V., "Congestion Avoidance and Control", Computer Communication Review, vol. 18, no. 4, pp. 314-329, Aug. 1988. ftp://ftp.ee.lbl.gov/papers/congavoid.ps.z.

[Jac90] Jacobson, V., "Modified TCP Congestion Avoidance Algorithm", end2end-interest mailing list, April 30, 1990. ftp://ftp.isi.edu/end2end/end2end-interest-1990.mail.

[MD90] Mogul, J. and S. Deering, "Path MTU Discovery", RFC 1191, November 1990. 
[MM96a] Mathis, M. and J. Mahdavi, "Forward Acknowledgment: Refining TCP Congestion Control", Proceedings of SIGCOMM' 96, August, 1996, Stanford, CA. Available

fromhttp://www.psc.edu/networking/papers/papers.html

[MM96b] Mathis, M. and J. Mahdavi, "TCP Rate-Halving with Bounding Parameters", Technical report. Available from http://www.psc.edu/networking/papers/FACKnotes/current.

[MMFR96] Mathis, M., Mahdavi, J., Floyd, S. and A. Romanow, "TCP Selective Acknowledgement Options", RFC 2018, October 1996.

[PAD+98] Paxson, V., Allman, M., Dawson, S., Fenner, W., Griner, J., Heavens, I., Lahey, K., Semke, J. and B. Volz, "Known TCP Implementation Problems", RFC 2525, March 1999.

[Pax97] Paxson, V., "End-to-End Internet Packet Dynamics", Proceedings of SIGCOMM '97, Cannes, France, Sep. 1997.

[Pos81] Postel, J., "Transmission Control Protocol", STD 7, RFC 793, September 1981 .

[Ste94] Stevens, W., "TCP/IP Illustrated, Volume 1: The Protocols", Addison-Wesley, 1994.

[Ste97] Stevens, W., "TCP Slow Start, Congestion Avoidance, Fast Retransmit, and Fast Recovery Algorithms", RFC 2001, January 1997 .

[WS95] Wright, G. and W. Stevens, "TCP/IP Illustrated, Volume 2: The Implementation", Addison-Wesley, 1995. 


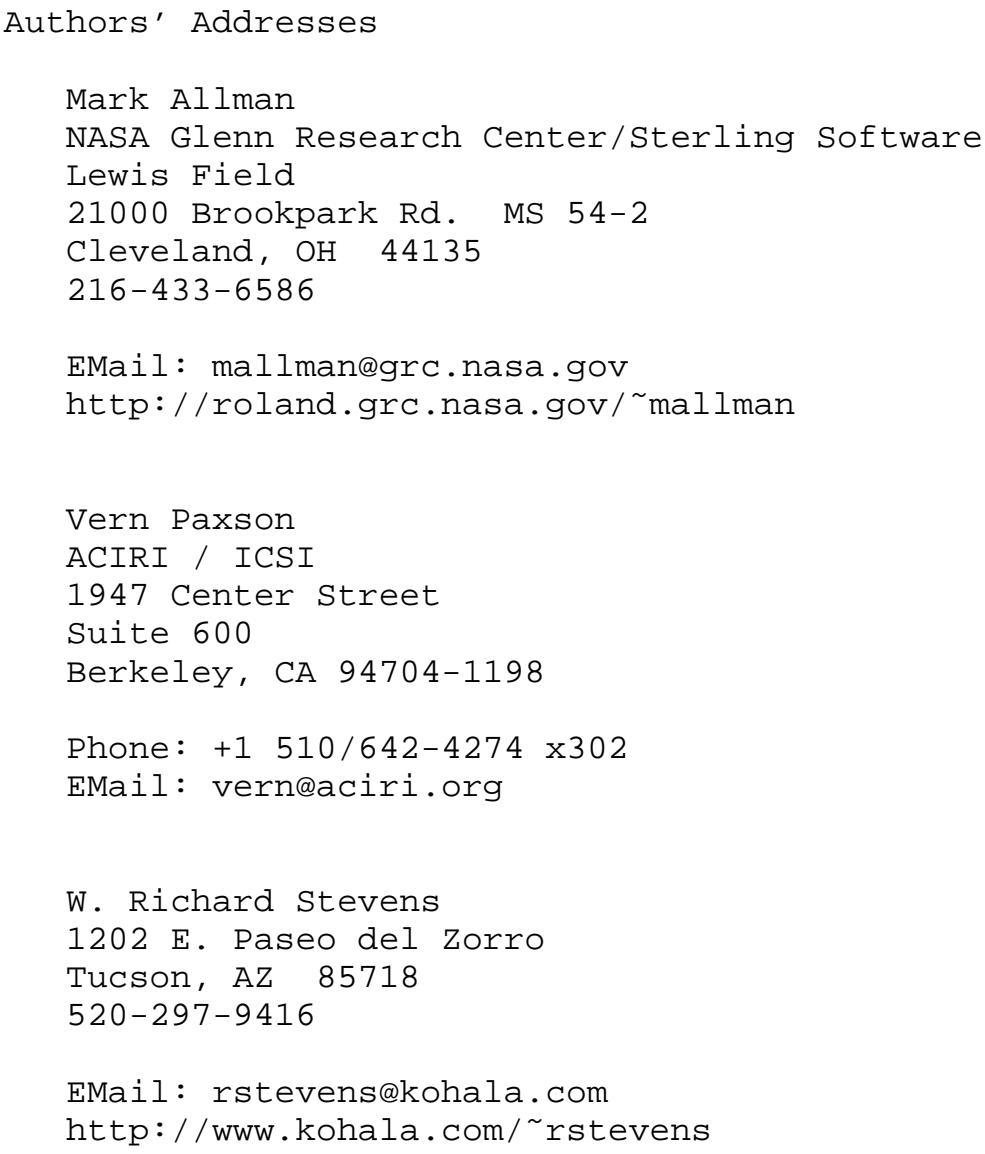


Full Copyright statement

Copyright (C) The Internet Society (1999). All Rights Reserved.

This document and translations of it may be copied and furnished to others, and derivative works that comment on or otherwise explain it or assist in its implementation may be prepared, copied, published and distributed, in whole or in part, without restriction of any kind, provided that the above copyright notice and this paragraph are included on all such copies and derivative works. However, this document itself may not be modified in any way, such as by removing the copyright notice or references to the Internet society or other Internet organizations, except as needed for the purpose of developing Internet standards in which case the procedures for copyrights defined in the Internet Standards process must be followed, or as required to translate it into languages other than English.

The limited permissions granted above are perpetual and will not be revoked by the Internet society or its successors or assigns.

This document and the information contained herein is provided on an "AS IS" basis and THE INTERNET SOCIETY AND THE INTERNET ENGINEERING TASK FORCE DISCLAIMS ALL WARRANTIES, EXPRESS OR IMPLIED, INCLUDING BUT NOT LIMITED TO ANY WARRANTY THAT THE USE OF THE INFORMATION HEREIN WILL NOT INFRINGE ANY RIGHTS OR ANY IMPLIED WARRANTIES OF MERCHANTABILITY OR FITNESS FOR A PARTICULAR PURPOSE. 